\title{
Biomechanics: a fundamental tool with a long history (and even longer future!)
}

\section{Bernardo Innocenti}

BEAMS Department (Bio Electro and Mechanical Systems), Université Libre de Bruxelles, Bruxelles, Belgium

\author{
Corresponding author: \\ Bernardo Innocenti \\ BEAMS Department (Bio Electro and Mechanical \\ Systems), \\ Université Libre de Bruxelles \\ Av. F. Roosevelt 50 \\ 1050 Bruxelles, Belgium \\ E-mail: bernardo.innocenti@ulb.ac.be
}

Biomechanics, (from Ancient Greek: Bios "life" and $\mu \eta \chi \alpha v \iota \kappa \dot{n}$ "mechanics"), is the application of mechanical principles to living organisms, such as humans, animals, plants and the functional basic units of life, the cells. It is now widely recognized that biomechanics plays an important role in the understanding of the fundamental principles of human motion; however, biomechanics is a field that has a very long history.

Already Aristotle (384-322 B.C.) in his book "De Motu Animalium" (On the Movement of Animals) defined animals' bodies as mechanical systems and he described the actions of the muscles and subjected them to geometric analysis for the first time. Archimedes (287-2I2 B.C.) developed principles which provided the basis for today's sport of swimming. Later, Leonardo da Vinci (1452-1519), considered an originator of this field, by studying anatomy in the context of mechanics, gained some insights into functional anatomy. Furthermore, he analyzed muscle forces as acting along lines connecting origins and insertions and studied joints function. Throughout the fifteenth, sixteenth, and seventeenth centuries, Galileo (1564-1642), Newton (16421726), and Borelli (1608-1679) gave their contribution to this field. In particular, Galileo analyzed the bone strength and shape change induced by an in- crease of weight and Newton published the famous laws of motion. Borelli was the first to determine the human joint force in equilibrium conditions and he also determined the position of the human center of gravity. During the 1800 and 1900 , revolutionary studies of musculoskeletal biomechanics were performed by Eadweard Muybridge, who used pioneering work in photography and the first motion-picture project to study human and animal motion; while, Julies Wolff postulated Wolff's law, which describes the relationship between trabecular bone geometries and mechanical stimuli on bone, that, based on observations in his long career as a surgeon. Then, in the 1960s, Al Burstein et al. began to teach biomechanical principles to orthopaedic surgeons.

Since then, the field of orthopaedic biomechanics has blossomed, producing significant works in the areas of biomechanics of bone, articular cartilage, soft tissues, upper extremities, spine, and so on. More sophisticated equipments and analyses have become available to perform advanced experiments that yielded much better understanding of joint kinematics and tissue function during walking, running and other activities of daily living. Mathematical modelling and improved engineering design of orthopaedic implants have also taken great strides. Bioengineers in collaboration with orthopaedic surgeons have applied biomechanical principles to study clinically relevant problems, improving patient treatments and outcomes.

It should be noted that biomechanists have provided very important information regarding movement patterns, joint force trend, and injury prevention and treatments, to name a few examples.

There are still many unanswered questions to be solved and a great deal of knowledge yet to be obtained. To let this discipline keep on growing, and enabling its place in tomorrow's society, we need to still move forward in research and education, interact together with all the players of this multidisciplinary field and bring our own contribution available for the future generations.

For this reason, this issue of Muscles, Ligaments and Tendons Journal focuses on basic and applied research in Biomechanics. You will find several different applications, using state of the art and innovative approaches and techniques, performed by different experts around the world. 
We understand that some of the topic could be challenging to be managed, but we believed that this issue could represent a great opportunity to stimulate the readers to approach the Biomechanics World, that is challenging, exciting, and, above all, funny... Enjoy!

Bernardo Innocenti 